\title{
Model of neutrino effective masses
}

\author{
Dinh Nguyen Dinh, ${ }^{1,2}$ Nguyen Anh Ky, ${ }^{1,2,3,4}$ Nguyen Thi Hong Van, ${ }^{1,2}$ and Phi Quang Van ${ }^{1}$ \\ ${ }^{1}$ Institute of Physics and Electronics, VAST, 10 Dao-Tan, Hanoi, Vietnam \\ ${ }^{2}$ Abdus Salam International Centre for Theoretical Physics, Strada Costiera 11, I-34014 Trieste, Italy \\ ${ }^{3}$ LAPTH, 9 Chemin de Bellevue, B.P. 110, F-74941 Annecy-le-Vieux Cedex France \\ ${ }^{4}$ Theory Devision, CERN, CH-1211 Geneva 23, Switzerland \\ (Received 22 May 2006; revised manuscript received 28 September 2006; published 26 October 2006)
}

It is shown that an effective (nonrenormalizable) coupling of lepton multiplets to scalar triplets in the 331 model with sterile/exotic neutrinos, can be a good way for generating neutrino masses of different types. The method is simple and avoids radiative/loop calculations which, sometimes, are long and complicated. Basing on some astrophysical arguments it is also stated that the scale of $S U(3)_{L}$ symmetry breaking is at $\mathrm{TeV}$ scale, in agreement with earlier investigations. Or equivalently, starting from this symmetry breaking scale we could have sterile/exotic neutrinos with mass of a few keV's which could be used to explain several astrophysical and cosmological puzzles, such as the dark matter, the fast motion of the observed pulsars, the re-ionization of the Universe, etc.

DOI: 10.1103/PhysRevD.74.077701

PACS numbers: 12.10.Dm, 12.60.Fr, 14.60.Pq

\section{INTRODUCTION}

Considered first by B. Pontecorvo [1] almost 50 years ago, the problems of neutrino oscillations and masses today are important not only for particle physics [2-12] but also for astrophysics and cosmology [2,7,9-11,13-15]. Different models and mechanisms of generating neutrino masses have been proposed (see, for instance, [2-12]) and various experiments $[9,10,16-24]$ have been organized in order to observe if neutrinos can oscillate and have masses. Although there are still open questions [8], many theoretical motivations and experimental results support the idea of massive neutrinos. It has not yet been clear, however, if the latter are of Dirac- or Majorana type or both. In the models with massive Dirac neutrinos only a certain combination of the lepton numbers can be a conserved quantity, while an existence of massive Majorana neutrinos (in processes like neutrinoless double beta $\left((\beta \beta)_{0 \nu}\right)$ decays [21-28]) violates it. In a recent work [29] we suggested a way of generating neutrino masses including Dirac and Majorana masses. It was done between an extension of Standard Model (SM), called also a 331 model, based on the gauge group $S U(3)_{C} \otimes S U(3)_{L} \otimes U(1)$. Neutrino mass terms arise via Yukawa couplings of lepton $S U(3)_{L}$-(anti)triplets with a scalar $S U(3)_{L}$-sextet (or shortly, sextet). This mass term was analyzed there [29] in different aspects, specially, at the seesaw limit [2-12,30] which might ensure neutrino masses at right orders. However, the Higgs sector of this 331 model also contains triplets under $S U(3)_{L}$. In this paper we will show how to use these triplets via their effective nonrenormalizable couplings with lepton multiplets to generate neutrino (effective) masses of a general structure. The results obtained may have astrophysical and cosmological applications. In the next section we briefly review the 331 model with two neutrinos in a lepton multiplet. The neutrino masses are considered in Sec. III. Finally, some comments and conclusions are given in Sec. IV.

\section{331 MODEL WITH STERILE/EXOTIC NEUTRINOS}

There are several versions of the 331 model [31-36] characterized with that the left-handed fermions (either leptons or quarks) of each generation are grouped in an $S U(3)_{L}$-(anti)triplet which has, besides two components of the corresponding SM $S U(2)_{L}$-doublet, an additional component being a sterile/exotic fermion or a chargeconjugation of a known right-handed fermion. The version considered in [29] (see also [32,35,36]) is the one in which the additional component of a lepton triplet could be a sterile/exotic neutrino $\left(n_{L}\right)$ which might be heavy, while the right-handed charged lepton $\left(l_{R}\right)$ still transforms as an $S U(3)_{L}$-singlet,

$$
f_{L}^{a}=\left(\nu_{L}^{a}, l_{L}^{a}, n_{L}^{a}\right)^{T} \sim(1,3,-1 / 3), \quad l_{R}^{a} \sim(1,1,-1),
$$

where $a$ is a generation index. Here we do not use the terminology "right-handed" but "sterilelexotic" as the helicity looses its absolute meaning when the particle to which it is appended, becomes massive.

The scalar sector of this model [29] contains three triplets

$$
\begin{aligned}
& \eta=\left(\eta_{1}^{0}, \eta_{2}^{-}, \eta_{3}^{0}\right)^{T} \sim(1,3,-1 / 3), \\
& \rho=\left(\rho_{1}^{+}, \rho_{2}^{0}, \rho_{3}^{+}\right)^{T} \sim(1,3,2 / 3), \\
& \chi=\left(\chi_{1}^{0}, \chi_{2}^{-}, \chi_{3}^{0}\right)^{T} \sim(1,3,-1 / 3),
\end{aligned}
$$

and a sextet

$$
\mathcal{S}=\left(\begin{array}{ccc}
\tau_{1}^{0} & T_{1}^{-} / \sqrt{2} & \tau_{2}^{0} / \sqrt{2} \\
T_{1}^{-} / \sqrt{2} & T_{2}^{--} & T_{3}^{-} \\
\tau_{2}^{0} / \sqrt{2} & T_{3}^{-} & \tau_{3}^{0}
\end{array}\right) \sim(1,6,-2 / 3)
$$

We observe that the Higgs fields $\eta$ and $\chi$ have the same charge structure and we may ask the question if we can discard one of them. Our answer is we may not be able to 
do that without imposing any physical constraints, as in general a VEV can be developed in three independent directions we need all these three triplets. The general form of the VEV's of these scalar fields is

$$
\begin{gathered}
\langle\eta\rangle=\left(u_{1}, 0, u_{3}\right)^{T}, \quad\langle\rho\rangle=\left(0, v_{2}, 0\right)^{T}, \\
\langle\chi\rangle=\left(w_{1}, 0, w_{3}\right)^{T} .
\end{gathered}
$$

The symmetry breaking hierarchy may give

$$
u_{3}, w_{3} \gg u_{1}, w_{1} \text {, }
$$

if $u_{3}$ and $w_{3}$ characterize the breaking scale of $S U(3)_{L}$, while $u_{1}$ and $w_{1}$ characterize the breaking scale of $S U(2)_{L}$. The condition (5) is logical as $u_{3}$ and $w_{3}$ can ensure large masses for exotic quarks (which should be very heavy) via Yukawa couplings of quark multiplets with $\eta$ and $\chi$. It is also consistent with the conditions minimizing the Higgs potential of, for example, the form

$$
\begin{aligned}
V= & \lambda_{1} \eta^{\dagger} \eta+\lambda_{2}\left(\eta^{\dagger} \eta\right)^{2}+\lambda_{3}\left(\eta^{\dagger} \chi+\chi^{\dagger} \eta\right)+\lambda_{4} \chi^{\dagger} \chi \\
& +\lambda_{5}\left(\chi^{\dagger} \chi\right)^{2} .
\end{aligned}
$$

The fields (2) may appear in some combinations such that their VEV configuration looks like

$$
\begin{gathered}
\left\langle\eta^{\prime}\right\rangle=\left(v_{1}, 0,0\right)^{T}, \quad\langle\rho\rangle=\left(0, v_{2}, 0\right)^{T}, \\
\left\langle\chi^{\prime}\right\rangle=\left(0,0, v_{3}\right)^{T} .
\end{gathered}
$$

which are linearly independent. In general, the VEV of the triplets has the structure (4) which in turn can be expressed in terms of (6) if the latter is taken for a basis.

As explained in [29], neutrino masses can be generated by coupling appropriate Higgs bosons to $\bar{f}_{L}\left(f_{L}\right)^{c}$. Being a tensor product $3^{*} \otimes 3^{*}$ of two $S U(3)_{L}$-anti-triplets, $\bar{f}_{L}$ and $\left(f_{L}\right)^{c}$, the latter can be decomposed into a direct sum of a triplet and an antisextet: $3^{*} \otimes 3^{*}=\mathbf{3} \oplus \mathbf{6}^{*}$. An $S U(3)_{L}$-invariant quantity can be built by contracting $\bar{f}_{L}\left(f_{L}\right)^{c}$ with an antitriplet $\mathbf{3}^{*}$ and/or a sextet $\mathbf{6}$. A contraction of the first kind can be $\lambda_{a b}^{\rho} \bar{f}_{L}^{a}\left(f_{L}^{b}\right)^{c} \rho^{\dagger}$ with $\lambda_{a b}^{\rho}$ being coupling constants. This (Yukawa) coupling term can generate Dirac masses for only two of the three neutrinos. This way of generating neutrino masses, however, gives no indication that they are small, and it excludes the Majorana neutrino masses which, as explained in [29], might be important (and natural to be considered since the neutrinos are neutral particles), specially, when the processes like $(\beta \beta)_{0 \nu}$ decays sensitive to the existence of Majorana neutrinos are still considered possible and intensively investigated [21-28]. Coupling of the sextet $\mathcal{S}$ to $\bar{f}_{L}\left(f_{L}\right)^{c}$,

$$
\lambda_{a b}^{s} \bar{f}_{L}^{a}\left(f_{L}^{b}\right)^{c} \mathcal{S}+\text { H.c., }
$$

(which is a contraction of the second kind) may solve this question [29]. It is shown in [29] that neutrino masses could be generated via this Yukawa coupling and the smallness of ordinary neutrinos and the largeness of the sterile/exotic neutrinos could be achieved via a seesaw limit of the mass matrix. In the next section we suggest one way more for generating neutrino masses.

\section{NEUTRINO EFFECTIVE MASSES VIA SCALAR TRIPLETS}

As said above, we can construct an $S U(3)_{L}$ invariant quantity by contracting $\bar{f}_{L}\left(f_{L}\right)^{c}$ with an antitriplet or a sextet. It can be done with the antitriplet $\rho \dagger$ and the sextet $\mathcal{S}$ [29]. There is, however, another way to have an antitriplet and a sextet to serve this goal. They can be constructed from the triplets $\eta$ and $\chi$ by taking the antisymmetric or the symmetric part of their tensor product, $3 \otimes 3=3^{*} \oplus \mathbf{6}$. In the matrix form the latter reads

$$
\eta \otimes \chi=\left(\begin{array}{c}
t_{1}^{-} \\
t_{2}^{0} \\
t_{3}^{-}
\end{array}\right) \oplus\left(\begin{array}{ccc}
s_{11}^{0} & s_{12}^{-} & s_{13}^{0} \\
s_{12}^{-} & s_{22}^{-} & s_{23}^{-} \\
s_{13}^{0} & s_{23}^{-} & s_{33}^{0}
\end{array}\right) \equiv T \oplus \Sigma,
$$

where

$$
\begin{gathered}
t_{1}^{-}=\left(\eta_{2}^{-} \chi_{3}^{0}-\eta_{3}^{0} \chi_{2}^{-}\right) / 2, \quad t_{2}^{0}=\left(\eta_{3}^{0} \chi_{1}^{0}-\eta_{1}^{0} \chi_{3}^{0}\right) / 2, \\
t_{3}^{-}=\left(\eta_{1}^{-} \chi_{2}^{0}-\eta_{2}^{0} \chi_{1}^{-}\right) / 2,
\end{gathered}
$$

and

$$
s_{i j}=\left(\eta_{i} \chi_{j}+\eta_{j} \chi_{i}\right) / 2, \quad i, j=1,2,3 .
$$

Contracting $T$ and $\Sigma$ with $\bar{f}_{L}\left(f_{L}\right)^{c}$ we get the effective (nonrenormalizable) couplings

$$
\begin{aligned}
& \mathcal{L}_{\text {(eff) }}^{\bar{f} f T}=G_{a b}^{T} \bar{f}_{L}^{a}\left(f_{L}^{b}\right)^{c} T+\text { H.c., } \\
& \mathcal{L}_{\text {(eff) }}^{\bar{f} f \Sigma}=G_{a b}^{\Sigma} \bar{f}_{L}^{a}\left(f_{L}^{b}\right)^{c} \Sigma+\text { H.c., }
\end{aligned}
$$

which are irreducible components decomposed from the nonrenormalizable four-particle interaction $\bar{f}_{L}\left(f_{L}\right)^{c} \eta \chi$, where $G_{a b}^{T, \Sigma}$ are effective coupling constants. Via these couplings, the antitriplet $T$ can give (effective) Dirac masses to two neutrinos only, whereas $\Sigma$ can offer neutrinos a richer (effective) mass structure.

The neutrino Dirac masses generated by (11) can be small if the effective coupling constants $G_{a b}^{T}$ are small (it is usually satisfied by radiative/loop induced $G_{a b}^{T}$ ) or/and the VEV $\left\langle t_{2}^{0}\right\rangle$ is small. One of the ways this VEV can get a small value is $u_{3} \sim w_{3}$ and $u_{1} \sim w_{1}$. These relations does not contradict with (5) where $u_{3}$ and $w_{3}$ are assumed to have a magnitude of the $S U(3)_{L}$-breaking scale, while $u_{1}$ and $w_{1}$-that of the $S U(2)_{L}$-breaking scale. If the VEV of the triplets has the structure (6) the condition for small neutrino masses is $G_{a b}^{T}$ to be small or/and $v_{1} v_{3}$ to be small. Some of the values of $u_{i}, v_{i}$ and $w_{i}$ may be also controlled by the masses of the charged leptons and quarks. Since, as said above, the exotic quarks must be heavy, $u_{3}$ and $w_{3}$ should be large. It is also important to consider if neutrinos have Majorana masses which could be generated by couplings like (12). Inserting the VEV 


$$
\langle\Sigma\rangle=\left(\begin{array}{ccc}
\left\langle s_{11}^{0}\right\rangle & 0 & \left\langle s_{13}^{0}\right\rangle \\
0 & 0 & 0 \\
\left\langle s_{13}^{0}\right\rangle & 0 & \left\langle s_{33}^{0}\right\rangle
\end{array}\right)
$$

of $\Sigma$ in (12) we get a mass term, which in the neutrino subspace can be written in the form of the standard DiracMajorana mass term,

$$
\frac{1}{2}\left(\bar{\nu}_{L}, \bar{n}_{L}\right)\left(\begin{array}{cc}
\mathbf{m}_{T} & \mathbf{m}_{D} \\
\mathbf{m}_{D} & \mathbf{m}_{S}
\end{array}\right)\left(\begin{array}{c}
\nu_{L}^{c} \\
n_{L}^{c}
\end{array}\right),
$$

with $\mathbf{m}_{T, D, S}$ being $3 \times 3$ matrices,

$$
\begin{gathered}
\left(\mathbf{m}_{T}\right)_{a b}=2 G_{a b}^{\Sigma}\left\langle s_{11}^{0}\right\rangle, \quad\left(\mathbf{m}_{D}\right)_{a b}=2 G_{a b}^{\Sigma}\left\langle s_{13}^{0}\right\rangle, \\
\left(\mathbf{m}_{S}\right)_{a b}=2 G_{a b}^{\Sigma}\left\langle s_{33}^{0}\right\rangle,
\end{gathered}
$$

where

$$
\begin{gathered}
\left\langle s_{11}^{0}\right\rangle=u_{1} w_{1}, \quad\left\langle s_{33}^{0}\right\rangle=u_{3} w_{3}, \\
\left\langle s_{13}^{0}\right\rangle=\frac{1}{2}\left(u_{1} w_{3}+u_{3} w_{1}\right) .
\end{gathered}
$$

At the type I seesaw limit (in fact, we compare the eigenvalues of the matrices),

$$
\mathbf{m}_{S} \gg \mathbf{m}_{D} \gg \mathbf{m}_{T} \approx 0,
$$

or equivalently, $\left\langle s_{33}^{0}\right\rangle \gg\left\langle s_{12}^{0}\right\rangle \gg\left\langle s_{11}^{0}\right\rangle \approx 0$, we get the eigen mass matrices (generation-mixing, in general)

$$
\mathbf{m}_{1}=-\left(\mathbf{m}_{D}\right)^{T}\left(\mathbf{m}_{S}\right)^{-1} \mathbf{m}_{D}, \quad \mathbf{m}_{2}=\mathbf{m}_{S},
$$

corresponding to two types of neutrinos, very light and very heavy, respectively. The conditions (17) could be satisfied if (5) is taken into account and one or both of $u_{1}$ and $w_{1}$ are approximately equal to zero (i.e., $u_{1} \approx 0$ or/and $w_{1} \approx 0$ ). At this seesaw limit the ration between the masses $\left(m_{1}\right)_{k}$ of the active (light) neutrinos and those $\left(m_{2}\right)_{k}$ of the sterile/exotic (heavy) neutrinos

$$
\frac{\left(m_{1}\right)_{k}}{\left(m_{2}\right)_{k}}=\left(\frac{\left\langle s_{13}^{0}\right\rangle}{\left\langle s_{33}^{0}\right\rangle}\right)^{2}, \quad k=1,2,3,
$$

does not depend on coupling constants. Taking (5) and (16) into account we can say that

$$
\left\langle s_{13}^{0}\right\rangle \sim \Lambda_{s u(2)} \cdot \Lambda_{s u(3)}, \quad\left\langle s_{33}^{0}\right\rangle \sim\left(\Lambda_{s u(3)}\right)^{2},
$$

where $\Lambda_{s u(2)}$ and $\Lambda_{s u(3)}$ are $S U(2)_{L^{-}}$and $S U(3)_{L}$ breaking scales, respectively. Therefore,

$$
\frac{\left(m_{1}\right)_{k}}{\left(m_{2}\right)_{k}} \sim\left(\frac{\Lambda_{s u(2)}}{\Lambda_{s u(3)}}\right)^{2} .
$$

It is popular to accept that the masses $m_{1}$ of the active neutrinos, if not zero, should be very small, while the masses $m_{2}$ of the sterile/exotic neutrinos could be very large. However, a not very heavy sterile/exotic neutrinos may be necessary. According to recent arguments, a mass $m_{2}$ of a few keV's may resolve several astrophysical and cosmological puzzles [37,38]. For example, such relatively light sterile/exotic neutrinos could account for cosmologi- cal dark matter, the origin of the large velocities of the observed pulsars and the re-ionization of the Universe [38]. So taking $m_{1} \sim 0.1-1 \mathrm{eV}$ and $m_{2} \sim 10^{3} \mathrm{eV}$ we get from (19) and (20)

$$
\frac{\Lambda_{s u(2)}}{\Lambda_{s u(3)}} \sim 10^{-1}-10^{-2} .
$$

It means a value of $\Lambda_{s u(3)}$ at a TeV scale which is in good agreement with earlier results obtained by other ways $[39,40]$. We can follow the inverse direction, that is, starting from a TeV $S U(3)_{L}$ symmetry breaking scale we can get a sterile/exotic neutrino mass at a few keV's which can be used to explain some astrophysical and cosmological problems mentioned above.

Next, we consider the type II seesaw limit (which was earlier applied to the left-right symmetry model $[7,41])$,

$$
\mathbf{m}_{S} \gg \mathbf{m}_{D} \gg \mathbf{m}_{T} \neq 0 .
$$

At this limit the eigen mass matrices are

$$
\mathbf{m}_{1}=\mathbf{m}_{\mathbf{T}}-\left(\mathbf{m}_{D}\right)^{T}\left(\mathbf{m}_{S}\right)^{-1} \mathbf{m}_{D}, \quad \mathbf{m}_{2}=\mathbf{m}_{S} .
$$

The limit (22) can be achieved by keeping (5) with both $u_{1}$, $w_{1} \neq 0$ such that $u_{1} w_{1}$ is still small. Since the latter may not be very small we get not so light active neutrinos, at the meantime the very large $\mathbf{m}_{\mathbf{S}}$ leads to very heavy sterile/ exotic neutrinos. In the other hand, the value of $\left(\mathbf{m}_{D}\right)^{T} \times$ $\left(\mathbf{m}_{S}\right)^{-1} \mathbf{m}_{D}$, small enough and close to that of $\mathbf{m}_{\mathbf{T}}$, provides a small $\mathbf{m}_{\mathbf{1}}$ as needed. In general, there is no constrain and correlation between $\mathbf{m}_{\mathbf{T}}$ and $\mathbf{m}_{\mathbf{S}}$.

As mentioned in [29], we can consider other limits of the mass term (14): the pure Majorana-, the Dirac- or the pseudo-Dirac limit, etc. They can be "translated" to the language of the scalar triplet VEV's, for instance, the Dirac limit can follow from the VEV configuration (6).

\section{CONCLUSION}

We have considered a scheme for generating neutrino masses between the 331 model with sterile/exotic neutrinos via effective (nonrenormalizable) couplings of lepton multiplets to Higgs triplets. The obtained mass term has a rich structure and it at some, e.g., the seesaw, limits, can generate neutrinos masses of different types at right orders, that is, small masses for ordinary (active) neutrinos and large masses for sterile/exotic neutrinos. Many theoretical models assume the latter to be very heavy, however, according to some recent arguments, sterile/exotic neutrinos with mass of a few keV's could help us to explain some astrophysical and cosmological puzzles. It is in agreement with earlier results stating that the $S U(3)_{L}$ symmetry breaking scale is at TeV's. The present work is one of the attempts to explain the nature of neutrino masses between the 331 model (see, for example, [27,42-48]). The advantage of this paper method is that it is simple and can estimate neutrino effective masses numerically without making radiative/loop calculations. 


\section{ACKNOWLEDGMENTS}

Three of us (D. N. D, N. A. K. and N. H. V.) would like to thank S. Randjbar-Daemi and the Abdus Salam ICTP, Trieste, Italy, for kind hospitality. One of us (N. A. K.) would like to thank P. Aurenche for kind hospitality at LAPTH, Annecy, France, and L. Alvarez-Gaume for kind hospitality at CERN, Geneva, Switzerland. Useful discussions with G. Belanger, J. Ellis, A. Kosenko, G. Senjanović and A. Smirnov are also acknowledged.

This work was supported in part by Vietnam National Program for Natural Sciences under Grant No 402106.
[1] B. Pontecorvo, JETP 6, 429 (1958); 7, 172 (1959).

[2] R. Mohapatra and P. Pal, Massive Neutrinos in Physics and Astrophysics (World Scientific, Singapore, 1998).

[3] F. Boehm and P. Vogel, Physics of Massive Neutrinos (Cambridge University Press, Cambridge, England, 1992).

[4] B. Kayser, F. Gibrat-Debu, and F. Perrier, The Physics of Massive Neutrinos (World Scientific, Singapore, 1989).

[5] R. Mohapatra and A. Smirnov, hep-ph/0603118.

[6] P. Langacker, Jens Erler and Eduardo Peinado, J. Phys.: Conf. Ser. 18, 154 (2005).

[7] R. Mohapatra et al., hep-ph/0510213.

[8] A. Smirnov, Int. J. Mod. Phys. A 19, 1180 (2004).

[9] S. Eidelman et al., Phys. Lett. B 592, 1 (2004); http:// pdg.lbl.gov.

[10] V. Barger, D. Marfatia, and K. Whisnant, Int. J. Mod. Phys. E 12, 569 (2003).

[11] J. Ellis, Nucl. Phys. B, Proc. Suppl. 91, 503 (2001).

[12] S. M. Bilenky and S. Petcov, Rev. Mod. Phys. 59, 671 (1987); 60, 575(E) (1988); 61, 169 (1989).

[13] J. Lesgourgues and S. Pastor, Phys. Rep. 429, 307 (2006).

[14] M. Fukugita, Nucl. Phys. B, Proc. Suppl. 155, 10 (2006).

[15] G. Raffelt, hep-ph/0208024.

[16] Y. Fukuda et al. (Super-Kamiokande Collaboration), Phys. Rev. Lett. 81, 1562 (1998); Phys. Lett. B 436, 33 (1998); 433, 9 (1998).

[17] W. Allison et al. (Soudan 2 Collaboration), Phys. Lett. B 449, 137 (1999).

[18] Q. Ahmad et al. (SNO Collaboration), Phys. Rev. Lett. 89, 011301 (2002); 89, 011302 (2002); V. Barger et al., Phys. Lett. B 537, 179 (2002); A. Baudyopadhyay et al., Phys. Lett. B 540, 14 (2002); J. Barcall et al., J. High Energy Phys. 07 (2002) 054; G. Fogli, E. Lisi, A. Marrone, D. Montanino, and A. Palazzo, Phys. Rev. D 66, 053010 (2002); B. Aharmim et al., Phys. Rev. C 72, 055502 (2005).

[19] C. Athanassopoulos et al. (LSND Collaboration), Phys. Rev. Lett. 81, 1774 (1998); Phys. Rev. C 58, 2489 (1998).

[20] H. Klapdor-Kleingrothaus et al. (Heidelberg-Moscow Collaboration), Nucl. Phys. B, Proc. Suppl. 110, 364 (2002); Phys. Lett. B 586, 198 (2004).

[21] Yu. Zdesenko, Rev. Mod. Phys. 74, 663 (2002).

[22] C. Aalseth et al., Phys. At. Nucl. 67, 2002 (2004).

[23] Ch. Kraus et al., Eur. Phys. J. C 40, 447 (2005).

[24] S. Choubey and W. Rodejohann, Phys. Rev. D 72, 033016 (2005).

[25] S. Bilenky, A. Faessler, T. Gutsche, and F. Simkovic, Phys. Rev. D 72, 053015 (2005).
[26] S. Petcov, Phys. Scripta T 121, 94 (2005).

[27] A. Dias, A. Doff, C. deS. Pires, and P. Rodrigues da Silva, Phys. Rev. D 72, 035006 (2005).

[28] S. Elliott and P. Vogel, Annu. Rev. Nucl. Part. Sci. 52, 115 (2002).

[29] Nguyen Anh Ky and Nguyen Thi Hong Van, Phys. Rev. D 72, 115017 (2005).

[30] M. Gell-Mann, P. Ramond, and R. Slansky, in Supergravity, edited by $\mathrm{P}$. van Nieuwenhuizen and D. Friedman (North Holland, Amsterdam, 1979), p. 315; R. Mohapatra and G. Senjanović, Phys. Rev. Lett. 44, 912 (1980).

[31] R. Foot, O. Hernandez, F. Pisano, and V. Pleitez, Phys. Rev. D 47, 4158 (1993).

[32] J. Montero, F. Pisano, and V. Pleitez, Phys. Rev. D 47, 2918 (1993).

[33] F. Pisano and V. Pleitez, Phys. Rev. D 46, 410 (1992).

[34] P. Frampton, Phys. Rev. Lett. 69, 2889 (1992).

[35] J. W. F. Valle and M. Singer, Phys. Rev. D 28, 540 (1983).

[36] M. Singer, J. W. F. Valle, and J. Schechter, Phys. Rev. D 22, 738 (1980).

[37] C. Watson, J. Beacom, H. Yüksel, and T. Walker, Phys. Rev. D 74, 033009 (2006); K. Abazajian and S. Koushiappas, Phys. Rev. D 74, 023527 (2006).

[38] P. Biermann and A. Kosenko, Phys. Rev. Lett. 96, 091301 (2006).

[39] Nguyen Anh Ky, Hoang Ngoc Long, and Dang Van Soa, Phys. Lett. B 486, 140 (2000); Nguyen Anh Ky and Hoang Ngoc Long, hep-ph/0103247.

[40] D. Ng, Phys. Rev. D 49, 4805 (1994).

[41] R. Mohapatra and G. Senjanović, Phys. Rev. D 23, 165 (1981); G. Lazarides, Q. Shafi, and C. Wetterich, Nucl. Phys. B181, 287 (1981).

[42] Nelson V. Cortez and Mauro D. Tonasse, Phys. Rev. D 72, 073005 (2005).

[43] A. Gusso, C. A. de S. Pires, and P. S. Rodrigues da Silva, Mod. Phys. Lett. A 18, 1849 (2003).

[44] T. Kitabayashi and M. Yasue, Phys. Lett. B 508, 85 (2001); Phys. Rev. D 63, 095002 (2001).

[45] J. Montero, C. deS. Pires, and V. Pleitez, Phys. Rev. D 65, 093017 (2002).

[46] J. Montero, C. deS. Pires, and V. Pleitez, Phys. Lett. B 502, 167 (2001).

[47] M. Tully and G. Joshi, Phys. Rev. D 64, 011301(R) (2001).

[48] Y. Okamoto and M. Yasue, Phys. Lett. B 466, 267 (1999). 